\title{
V. On magnetic lag
}

\section{Thomas H. Blakesley M.A.}

To cite this article: Thomas H. Blakesley M.A. (1888) V. On magnetic lag , Philosophical Magazine Series 5, 26:158, 34-43, DOI: $10.1080 / 14786448808628233$

To link to this article: http://dx.doi.org/10.1080/14786448808628233

$$
\text { 册 Published online: } 29 \text { Apr } 2009 .
$$

Submit your article to this journal $2 \pi$

Q View related articles ¿ 


\section{[ 34 ] \\ V. On Magnetic Lag. \\ By Thomas H. Blakesley, M.A.*}

TN bringing my views on Transformers before the Physical Society it is my desire to emphasize :-

(1) How the magnetic lag, if it exist, may be measured by employing dynamometers of low resistance.

(2) That the magnetic lag has a real existence.

(3) That the magnetic lag necessarily accompanies an absorption of work involved in the reversal of polarity in the iron, and how this may be measured.

(4) The points in the general argument where scientific facts are wanting, and the direction which investigation should take to meet this want.

The possibility of the existence of a magnetic lag renders the problem a different one from that of two coils acting and reacting upon themselves by means of mutual and selfinduction, whose coefficients, being geometrical, are constant.

For the latter problem I gave in the year 1885 a complete solution, but I pointed out that the completeness of the result rested upon the absence of anything in the nature of hysteresis (a word not then in use) or work done in the field.

The following year Mr. George Forbes, F.R.S., gave what should have been (but for the very poor reporting of the Society of Arts' Journal) a solution of the " secondary generator" problem, treating it as a case of two coils, assuming that "the magnetism of the core varies as the sum of the currents in the two coils;" and the same gentleman has treated the subject again in a recent paper before the Society of TelegraphEngineers and Electricians, in which he makes the same assumption, and says, referring to the harmonic functions which he attributes to the electrical and magnetic quantities involved, that the existence of magnetic hysteresis would cause departure from the harmonic character, but that, being insignificant so long as the magnetic induction in the iron is not high, its consideration may be omitted; statements which seem rather to evade than to overcome the difficulty.

Mr. Gisbert Kapp, who has done so much good work in the practical development of transformers, also, in my opinion, makes the same assumption, that the state of magnetization in the core coincides with the magnetic stress resulting from compounding the stresses derived from the two coils.

* Communicated by the Physical Society : read May 12, 1888. 
In the view I shall put forward I shall assume :-

(1) That the variations are harmonic.

(2) That the only induction in the secondary coil is derived from the core, and is therefore, as regards phase, in quadrature with the magnetization. As the current in the secondary coil will be considered as producing one of the components of the stress producing magnetization, itself reacting upon the coil, the necessity of introducing a special E.M.F. of self-induction is obviated.

(3) That each turn in either coil embraces the same number of magnetic lines.

I shall also make use of the following symbols :-

$\mathrm{E}$, the maximum electromotive force of the machine ;

$I_{1}$, the maximum value of the current in the primary circuit ;

$\mathrm{I}_{2}$, the maximum value of the current in the secondary circuit ;

$\pi-\theta$, the angle of phase-difference between the currents;

$m$, the number of turns of wire in the primary coil ;

$n$, $, \quad, \quad, \quad$ secondary coil ;

$\phi$, the angle of magnetic lag";

$r_{1}$, the resistance in the primary circuit;

$r_{2}, \quad, \quad, \quad$ secondary circuit ;

$\left.\alpha_{1}\right\}$ are the readings of two dynamometers placed respectively in the primary and secondary circuits, their constants being $A$ and $B$, so that $\frac{I_{1}{ }^{2}}{2}=A \alpha_{1}, \frac{I_{2}{ }^{2}}{2}=B \alpha_{2}$;

$\alpha_{3}$ is the reading of a dynamometer one of whose coils is in the primary, the other in the secondary circuit. Its constant is $\mathrm{C}$.

$M$ is the maximum magnetization.

The magnetic stress produced by each coil is proportional to the current in that coil multiplied by the number of turns in the coil, and is here taken to be that product, called very often the ampere-turns. Its maximum value in the primary coil is $m \mathrm{I}_{1}$ and in the secondary coil it is $n \mathrm{I}_{2}$.

Now the observations on the dynamometers A and B furnish us with a knowledge of $\mathrm{I}_{1}$ and $\mathrm{I}_{2}$ in any case; and $m$ and $n$ are details of the construction of the transformer. Thus we are in possession of the two quantities $m \mathrm{I}_{1}$ and $n \mathrm{I}_{2}$.

But the three dynamometer observations enable us to determine the angle of phase-difference between the currents, as D 2 
I have elsowhere explained :-

$$
\cos \theta=\frac{\mathrm{C} \alpha_{3}}{\sqrt{\mathrm{A} \alpha_{1} \mathrm{~B} \alpha_{2}}} \text { for } \mathrm{C} \alpha_{3}=\frac{\mathrm{I}_{1} \mathrm{I}_{2} \cos \theta}{2} \text {. }
$$

We are therefore in possession of the two components of the magnetic stress and of the angle between them. Hence we are virtually in possession of the whole magnetic stress, and its phase relatively to its components. If the resultant is in quadrature with that component which results from the current in the secondary coil, it is in the same phase as the magnetization, which is in quadrature with that component; but not unless this is the case.

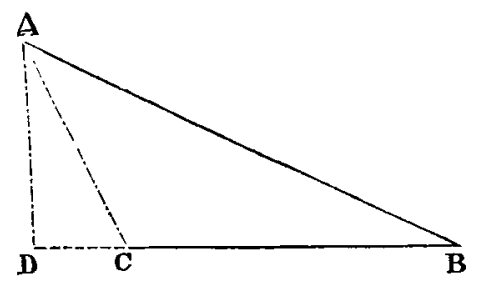

Let the line A B represent $m \mathrm{I}_{1}$ or the magnetic stress in the primary circuit, and let $\mathrm{BC}$ represent the magnetic stress in the secondary, and let A B C be the angle $\theta$, found as above. Then $A C$ is the resultant magnetic stress.

But the magnetization is in quadrature with $\mathrm{B} \mathrm{C}$. Draw $A D$ at right angles to $B C$. Then $C A D$ represents the magnetic lag, which is seen to vanish if $\mathrm{A} \mathrm{C} \mathrm{B}$ is a right angle. The condition of the existence of lag is therefore that

$$
\mathrm{CB}<\mathrm{AB} \cos \theta \text {, }
$$

which in terms of the dynamometer observations is

$$
n \sqrt{2 \mathrm{~B} \alpha_{2}}<m \sqrt{2 \mathrm{~A} \alpha_{1}} \frac{\mathrm{C} \alpha_{3}}{\sqrt{\mathrm{A} \alpha_{1} \mathrm{~B} \alpha_{2}}},
$$

or

$$
\mathrm{B} \alpha_{2}<\frac{m}{n} \mathrm{C} \alpha_{3} \text {. }
$$

The observation on the dynamometer in the primary is seen to be eliminated. Thus this question can be tested with two dynamometers only. The amount of lag is represented by the angle CAD. We can easily express its tangent in terms of the three dynamometer observations. 


$$
\begin{aligned}
\tan \phi=\frac{\mathrm{CD}}{\mathrm{DA}} & =\frac{\mathrm{DB}-\mathrm{BC}}{\mathrm{AB} \sin \theta}=\frac{\frac{\mathrm{DB}}{\mathrm{AB}}-\frac{\mathrm{BC}}{\mathrm{AB}}}{\sqrt{1-\cos ^{2} \theta}} \\
& =\frac{\cos \theta-\frac{n I_{2}}{m I_{1}}}{\sqrt{1-\cos ^{2} \bar{\theta}}} \\
& =\frac{\frac{\mathrm{C} \alpha_{3}}{\sqrt{\mathrm{A} \alpha_{1} \mathrm{~B} \alpha_{2}}}-\frac{n}{m} \sqrt{\frac{\overline{\mathrm{B} \alpha_{2}}}{\mathrm{~A} \alpha_{1}}}}{\sqrt{1-\frac{\mathrm{C}^{2} \alpha_{3}^{2}}{\mathrm{~A} \alpha_{1} \mathrm{~B} \alpha_{2}}}} \\
& =\frac{\mathrm{C} \alpha_{3}-\frac{n}{m} \mathrm{~B} \alpha_{2}}{\sqrt{\mathrm{A} \alpha_{1} \mathrm{~B} \alpha_{2}-\mathrm{C}^{2} \alpha_{3}{ }^{2}}}
\end{aligned}
$$

Thus the angle of magnetic lag, if it exist, can be detected with two dynamometers and measured with three.

In dealing yet further with the results furnished by the observations, we must remember that the waxing magnetism has the same inductive effect in producing E.M.F. in each turn of the coils of the two circuits. But we can, from the observation of dynamometer B, say what that E.M.F. per turn is. The whole E.M.F. in the secondary coil is $\mathrm{I}_{2} r_{2}$, consequently the E.M.F. per turn is $\frac{\mathrm{I}_{2} r_{2}}{n}$. Therefore in the primary the total E.M.F. arising from magnetic induction is $m \frac{\mathrm{I}_{2} r_{2}}{n}$.

The current arising from the same source is $\frac{m I_{2}}{n} \frac{r_{2},}{r_{1}}$ and the magnetizing stress on this account is $\frac{m^{2}}{n} \frac{\mathrm{I}_{2} r_{2}}{r_{1}}$, which must be looked upon as one of the components of the whole magnetizing stress due to the primary current; and this component is in the same phase as the magnetizing stress in the secondary.

Hence, returning to the figure, if we produce $\mathrm{CB}$ to $\mathrm{F}$ so that $\mathrm{CB}: \mathrm{BF}:: n \mathrm{I}_{2}: \frac{m^{2}}{n} \frac{\mathrm{I}_{2} r_{2}}{r_{1}}$,

$$
:: 1: \frac{m^{2}}{n^{2}} \frac{r_{2}}{r_{1}}
$$

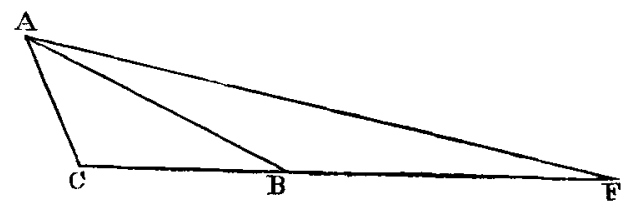


$\mathrm{FB}$ is one of the components of AB.

The other component ( $i$. e. that arising from the machine's proper electromotive force) is $\mathrm{AF}$. Hence $\mathrm{AF}=m \underset{r_{1}}{\mathrm{E}}$.

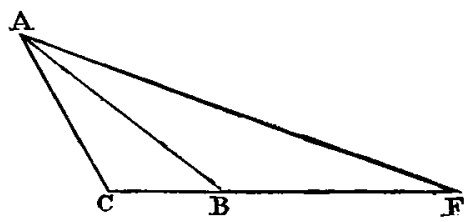

It follows that the electromotive force $\mathrm{E}$, which the machine is exerting, may be thus determined by means of the dynamometer observations.

$$
\begin{aligned}
& \mathrm{AF}^{2}=\mathrm{AB}^{2}+\mathrm{BF}^{2}+2 \mathrm{AB} \mathrm{BF} \cos \mathrm{ABC}, \\
& \frac{m^{2} \mathrm{E}^{2}}{r_{1}^{2}}=m^{2} \mathrm{I}_{1}^{2}+\left(\frac{m^{2}}{n^{2}} \frac{r_{2}}{r_{1}}\right)^{2} n^{2} \mathrm{I}_{2}^{2}+2 m \mathrm{I}_{1} \frac{m^{2}}{n^{2}} \frac{r_{2}}{r_{1}} n \mathrm{I}_{2} \cos \theta, \\
& \therefore \mathrm{E}^{2}=r_{1}^{2} \mathrm{I}_{1}^{2}+r_{2}{ }^{2} \mathrm{I}_{2}{ }^{2} \frac{m^{2}}{n^{2}}+2 r_{1} r_{2} \frac{m}{n} \mathrm{I}_{1} \mathrm{I}_{2} \cos \theta, \\
&=r_{1}^{2} 2 \mathrm{~A} \alpha_{1}+\frac{m^{2}}{n^{2}} r_{2}^{2} 2 \mathrm{~B} \alpha_{2}+4 r_{1} r_{2} \frac{m}{n} \mathrm{C} \alpha_{3}, \\
&=2\left\{r_{1}^{2} \mathrm{~A} \alpha_{1}+r_{2}{ }^{2} \frac{m^{2}}{n^{2}} \mathrm{~B} \alpha_{2}+2 r_{1} r_{2} \frac{m}{n} \mathrm{C} \alpha_{3}\right\} .
\end{aligned}
$$

Another interesting magnitude is AC, or the total impressed magnetic force.

$$
\begin{aligned}
\mathrm{AC}^{2} & =\mathrm{AB}^{2}+\mathrm{BC}^{2}-2 \mathrm{AB} \mathrm{BC} \cos \theta, \\
& =m^{2} \mathrm{I}_{1}^{2}+n^{2} \mathrm{I}_{2}^{2}-2 m n \mathrm{I}_{1} \mathrm{I}_{2} \cos \theta, \\
& =2 m^{2} \mathrm{~A} \alpha_{1}+2 n^{2} \mathrm{~B} \alpha_{2}-4 m n \mathrm{C} \alpha_{3}, \\
& =2\left\{m^{2} \mathrm{~A} \alpha_{1}+n^{2} \mathrm{~B} \alpha_{2}-2 m n \mathrm{C} \alpha_{3}\right\} .
\end{aligned}
$$

By means of this we may calculate what current should be passed through the primary circuit, the secondary being open, to produce the same state in the core.

But perhaps the most interesting point to men of science and to civil engineers is the question of power. We may approach it thus perhaps in the simplest way.

By dropping a perpendicular from $\mathrm{F}$ upon $\mathrm{AB}$ produced we easily see that

$A F \cos B A F=B F \cos A B C+A B$.

Multiplying through by $A B$ we have

$$
A F A B \cos B A F=A B B F \cos A B C+A^{2} \text {. }
$$




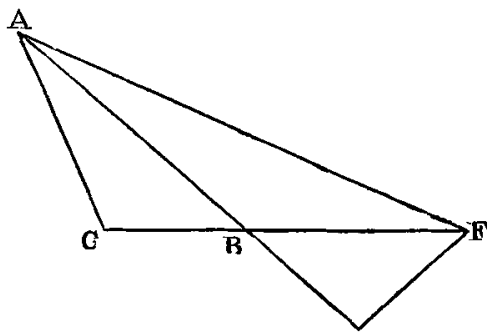

Substituting the electric quantities for the geometrical,

$$
\frac{m \mathrm{E}}{r_{1}} m \mathrm{I}_{1} \cos \mathrm{BAF}=m \mathrm{I}_{1} \frac{m^{2}}{n^{2}} \frac{r_{2}}{r_{1}} n \mathrm{I}_{2} \cos \theta+n^{2} \mathrm{I}_{1}^{2} ;
$$

multiply through by $\frac{r_{1}}{2 m^{2}}$,

$$
\frac{E I_{1} \cos \mathrm{BAF}}{2}=r_{2} \frac{m}{n} \frac{\mathrm{I}_{1} \mathrm{I}_{2} \cos \theta}{2}+\frac{r_{1} \mathrm{I}_{1}^{2}}{2} \text {. }
$$

But the term on the left is the expression for the total power, and those on the right hand may be expressed in terms of the dynamometer observations.

Thus the total power

$$
=r_{1} \mathrm{~A} \alpha_{1}+r_{2} \frac{m}{n} \mathrm{C} \alpha_{3} \text {. }
$$

The first term here is obviously the power at work heating the primary coil.

$r_{2} \mathrm{Ba}_{2}$ is as obviously the power heating the secondary coil. If, therefore, we write the total power

$$
=r_{1} \mathrm{~A} \alpha_{1}+r_{2} \mathrm{~B} \alpha_{2}+r_{2}\left\{\frac{m}{n} \mathrm{C} \alpha_{3}-\mathrm{B} \alpha_{2}\right\} \text {, }
$$

we see that the power involved with the magnetic lag is

$$
r_{2}\left\{\frac{m}{n} \mathrm{C} \alpha_{3}-\mathrm{B} \alpha_{2}\right\},
$$

the form showing that it disappears if the lag does so.

Thus we are led to the conclusion that a magnetic lag involves a loss of power, and any loss of power due to molecular action in the core taking place in the course of the alternations of magnetization must necessarily produce lag.

Now if the changing magnetization does work it must do it against a force, and this force must be of the character which of itself would produce magnetization, i.e. magnetic 
force. Just as when a body moving in a medium does work in the medium, it does so by calling into being, or inducing, a force, viz. friction; force being that sort of magnitude which acting upon a body produces motion.

By analogy alone, therefore, we may infer that when changing magnetization is a continuous source of absorbed work, the changing magnetization induces what would itself produce magnetization, that is an induced stress acting in opposition to the direction of the change in the magnetization.

This may perhaps be allowed; but it may be urged that there is plenty of magnetic stress already, impressed by the currents; why should not the changing magnetization work on this? The answer to this objection is that if there be no other stress but that impressed from outside, then the phase of the magnetization will be in the same phase, and therefore the increase of magnetization will be in quadrature with the stress, and hence no work will be continuously absorbed. For though through some phases work may be done in such a case, this is always recoverable and recovered in a complete period; a proposition which I have stated and proved in my papers upon Alternating Currents in 1885.

$I$ apprehend, therefore, that besides the stresses $\mathrm{AB} B C$ we have another induced stress in quadrature with the magnetization, because called into being by its increase, and therefore in the same phase as $\mathrm{FB}$ or $\mathrm{BC}$.

Let $\mathrm{BC}$, therefore, be produced until it meets in $\mathrm{D}$ the line $\mathrm{AD}$ drawn at right angles to $\mathrm{BC}$.

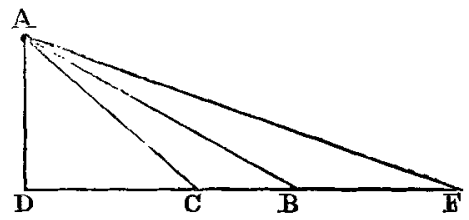

Then AD will be the effective magnetic stress, $i . e$. that magnetic stress which, maintained with a steady current, will produce the actual magnetization; hence, if $\mathrm{M}$ is the maximum magnetization,

$$
\frac{4 \pi \mathrm{AD}}{\rho}=\mathrm{M}
$$

where $\rho$ is called the magnetic resistance. Thus

$$
\mathrm{AD}=\frac{\mathrm{M}_{\rho}}{4 \pi}
$$


If $2 \mathrm{~T}$ is the period of alternation, $\frac{\pi \mathrm{M}}{\mathrm{T}}$ is the maximum rate of increase of $M$. If

$$
\mathrm{N} \frac{\pi \mathrm{M}}{\mathrm{T}}=\mathrm{DC}
$$

$\mathrm{N}$ may be called the coefficient of magnetic self-induction.

Under the exigencies of a certain temporary nomenclature CD might be called the Foucault-Ampere turns existing in the core itself.

Substituting for $\mathrm{M}$ in terms of $\mathrm{AD}$,

or

$$
\begin{gathered}
D C=\frac{N \pi}{\mathrm{T}} \frac{4 \pi \mathrm{AD}}{\rho}, \\
\frac{\mathrm{DC}}{\mathrm{AD}}=\tan \phi=\left\{\frac{4 \pi^{2}}{\mathrm{~T} \rho}\right\} \mathrm{N} .
\end{gathered}
$$

I have explained above how $\tan \phi$ may be obtained from the dynamometer observations. We may therefore employ this formula for the determination of the value and constancy of $\mathrm{N}$, if we can rely upon the values of $\mathrm{T}$ obtained by observation at the time, and of $\rho$ known otherwise.

The constancy of $\rho$ obtains so long as magnetization can keep pace with magnetic stress. These conditions are fairly well understood, and $A D$ must not exceed the stress representing the limiting one. Thus it will be well to know AD

$$
\begin{aligned}
\mathrm{AD}=\mathrm{AB} \sin \theta & =m \mathrm{I}_{1} \sqrt{1-\cos ^{2} \theta} \\
& =m \sqrt{2 \mathrm{~A} \alpha_{1}} \sqrt{1-\frac{\mathrm{C}^{2} \alpha_{3}^{2}}{\mathrm{~A} \alpha_{1} \mathrm{~B} \alpha_{2}}}, \\
& =\sqrt{\overline{2}} \cdot m \cdot \frac{\sqrt{\mathrm{A} \alpha_{1} \mathrm{~B} \alpha_{2}-\mathrm{C}^{2} \alpha_{3}{ }^{2}}}{\sqrt{\overline{\mathrm{B}} \alpha^{2}}}
\end{aligned}
$$

I think, therefore, experiment should move in the following direction. The constancy or the reverse of $\mathrm{N}$ under varying speeds should be first determined by experiments with currents so small or coils so few that the magnetic resistance $\rho$ may be safely assumed constant. For this purpose it would be necessary to employ some speed-indicator.

Professor Fortes, F.R.S., in his paper upon transformers, already quoted, says that the existence of hysteresis would cause a departure from the harmonic character of the motion, but that the effects are small and negligible.

If $\mathrm{N}$, however great, remains constant, the harmonic character of the variation is maintained. But if experience 
showed that induced stress varies as the square or higher power of the rate of change of magnetization, then indeed a serious modification would take place, and this would be likely if the neighbourhood of saturation were reached.

In the foregoing investigation I have represented magnetic stress as of the order Current, and the formulæ I have introduced hitherto will bear this convenient form of expression; but strictly speaking it is of the order Field, and when we wish to settle the dimensions of $\mathrm{N}$ we must take this into account. The $\mathbf{M}$ of the work is really of the order Moment or $\left[l^{\frac{5}{2}} t^{-1} m^{\frac{1}{2}}\right]$. Now the rate of increase of Moment multiplied into $\mathrm{N}$ is equal to Field or $\left[l^{-\frac{1}{2}} t^{-1} m^{\frac{1}{2}}\right]$;

$$
\begin{gathered}
\therefore \mathrm{N} \frac{\left[l^{\frac{5}{2}} t^{-1} m^{\frac{1}{2}}\right]}{t}=\left[l^{-\frac{1}{2}} t^{-1} m^{\frac{1}{2}}\right], \\
\therefore \mathrm{N}=\left[l^{-3} t\right] .
\end{gathered}
$$

The actual experiments, which I now bring to the notice of the Society, were carried out at the works of Messrs. Nalder, Brothers \& Co., of Westminster, and I am greatly indebted to these gentlemen themselves, and to Mr. Crawley and Mr. Mott for their assistance in making them, for they freely placed their steam-power, their electrical power, and their intellectual power at my disposal.

These experiments had no other object than to test the question of the existence of magnetic lag by dynamometers, and to measure the angle of lag.

The machine employed and the transformer were of the Kapp forms. The numbers of turns of wire in the two coils had been ascertained for me by Mr. Crawley. They were 100 and 12 respectively.

Considering the considerable differences in the relations of the currents, the constancy of the angle of lag appears to me to point to a simple law connecting it with the magnetization.

There was no very accurate speed-indicator employed, and the speed was approximately the same in the first six experiments. In the seventh experiment the speed was purposely much diminished, by about one third of that in the former cases, and in this experiment alone does the lag appear at less than $5^{\circ}$. 
On Electromotive Forces of Contact.

\begin{tabular}{|c|c|c|c|c|c|c|c|}
\hline $\begin{array}{l}\text { No. of } \\
\text { Experi- } \\
\text { ment. }\end{array}$ & $\begin{array}{c}A \alpha_{1}= \\
\frac{I_{2}^{2}}{2} .\end{array}$ & $\begin{array}{l}\mathrm{B} x_{2}= \\
\frac{\mathrm{I}_{2}^{2}}{2} .\end{array}$ & $\mathrm{C} \alpha_{3}$. & $\frac{m}{n} \mathrm{C}_{\boldsymbol{x}_{3}}$ & $\theta$. & $\phi$. & $\sqrt{\Delta \alpha_{1}} \sin \theta$ \\
\hline $1 . \ldots \ldots$ & $33 \cdot 29$ & $52 \cdot 65$ & $10 \cdot 37$ & $86 \cdot 46$ & $75^{\circ} 40^{\prime}$ & $5^{\circ} 43^{\prime}$ & $5 \cdot 590$ \\
\hline 2. ...... & $34 \cdot 43$ & 5974 & 11.50 & $95 \times 83$ & $\begin{array}{ll}75 & 19\end{array}$ & 538 & $5 \cdot 676$ \\
\hline 3. ...... & 37.09 & $92 \cdot 14$ & $17 \cdot 34$ & 144.50 & 7245 & 625 & $5 \cdot 816$ \\
\hline 4. ...... & $70 \cdot 38$ & 86.06 & $17 \cdot 43$ & $145 \cdot 22$ & 774 & 521 & $8 \cdot 176$ \\
\hline $5 . \ldots \ldots$ & $81 \cdot 17$ & $81 \cdot 00$ & $17 \cdot 21$ & $143 \cdot 42$ & 7745 & 524 & 8.804 \\
\hline 6. ...... & 84.97 & $29 \cdot 03$ & $7 \cdot 83$ & $65 \cdot 26$ & 8056 & $\begin{array}{ll}5 & 4\end{array}$ & $9 \cdot 103$ \\
\hline 7........ & $8 \cdot 21$ & $101 \cdot 59$ & $14 \cdot 31$ & $119 \cdot 26$ & $\begin{array}{ll}60 & 17\end{array}$ & 450 & $2 \cdot 488$ \\
\hline $\begin{array}{l}\text { No. of } \\
\text { Experi- } \\
\text { ment. }\end{array}$ & $r_{\lambda}$ & $r_{2}$ & $\frac{\mathrm{I}_{1}{ }^{2}}{2} r_{1}$ & $\frac{I_{2}^{2}}{2} r_{2^{*}}$ & \multicolumn{2}{|c|}{$\begin{array}{c}\text { Power of } \\
\text { internal } \\
\text { magnetic } \\
\text { work } \equiv \mathrm{H} .\end{array}$} & $\frac{H}{\Delta \alpha_{1} \sin ^{2} \theta}$. \\
\hline $1 . \ldots \ldots$ & $2 \cdot 01$ & $2 \cdot 51$ & $66 \cdot 91$ & $132 \cdot 15$ & \multicolumn{2}{|c|}{84.96} & 272 \\
\hline $2, \ldots \ldots$ & $"$ & $2 \cdot 36$ & $69 \cdot 20$ & 140.99 & \multicolumn{2}{|c|}{$85 \cdot 17$} & $2 \cdot 64$ \\
\hline 3....... & " & $1 \cdot 96$ & 7455 & $180 \cdot 59$ & \multirow{2}{*}{\multicolumn{2}{|c|}{$102 \cdot 63$}} & $\Im 03$ \\
\hline $4 \ldots \ldots$ & ", & $\ldots$ & $141 \cdot 46$ & & & & \\
\hline $5 \ldots \ldots$ & $"$ & $2 \cdot 87$ & $163 \cdot 15$ & $232 \cdot 47$ & \multicolumn{2}{|c|}{$179 \cdot 15$} & $2 \cdot 31$ \\
\hline $6, \ldots \ldots$ & ", & 477 & 17079 & $138 \cdot 47$ & \multicolumn{2}{|c|}{$172 \cdot 82$} & $2 \cdot 09$ \\
\hline $7 \ldots \ldots$ & $"$ & 0.72 & $16 \cdot 50$ & $73 \cdot 14$ & \multicolumn{2}{|c|}{$12 \cdot 73$} & $2 \cdot 06$ \\
\hline
\end{tabular}

VI. On Electromotive Forces of Contact. By Charles V. Burton, B.Sc.*

THE principal object of this paper is to discuss the seats 1 of the electromotive forces developed by the contact of conductors, and by considering the transformations of energy which occur when two insulated conductors are brought into contact, an attempt is made to give a logical demonstration of some of the views put forward. In the latter part of the paper a description is given of some experiments on the nature of the electrification of non-conductors by contact or friction.

When two conductors are brought into contact, an electromotive force (E) is set up between them, which drives

* Communicated by the Physical Society : read April 28, 1888. 